\title{
VARIABLE-THICKNESS BOUNDARY LAYERS FOR SLIDING MODE CONTROL
}

Chyun-Chau Fuh

Department of Mechanical and Mechatronic Engineering, National Taiwan Ocean University, Keelung, Taiwan, R.O.C., f0005@mail.ntou.edu.tw

Follow this and additional works at: https://jmstt.ntou.edu.tw/journal

Part of the Mechanical Engineering Commons

\section{Recommended Citation}

Fuh, Chyun-Chau (2008) "VARIABLE-THICKNESS BOUNDARY LAYERS FOR SLIDING MODE CONTROL," Journal of Marine Science and Technology. Vol. 16: Iss. 4, Article 7.

DOI: $10.51400 / 2709-6998.2014$

Available at: https://jmstt.ntou.edu.tw/journal/vol16/iss4/7

This Research Article is brought to you for free and open access by Journal of Marine Science and Technology. It has been accepted for inclusion in Journal of Marine Science and Technology by an authorized editor of Journal of Marine Science and Technology. 


\title{
VARIABLE-THICKNESS BOUNDARY LAYERS FOR SLIDING MODE CONTROL
}

\author{
Chyun-Chau Fuh*
}

Key words: sliding mode control, chattering, boundary layers.

\begin{abstract}
Sliding mode control is a nonlinear feedback control technique which provides good robustness against modeling uncertainties and external disturbances. However, as the uncertainties or disturbances increase, a greater control input is required, and the control signal may cause an undesirable chattering phenomenon. In general, the further the system trajectory from the origin, the more violent the chattering effect. However, as the trajectory moves closer to the origin, the degree of chattering gradually reduces. As a result, even if an attempt is made to eliminate chattering by introducing a small constant boundary layer around the sliding surface, the chattering phenomenon may still take place during the transient stage. Furthermore, if the thickness of the boundary layer is increased in order to suppress chattering completely, control accuracy may be lost. Accordingly, this study proposes four different variable-thickness boundary layers designed to achieve a compromise between the desire to eliminate the chattering phenomenon and the need to retain control accuracy. In two of these boundary layers, the thickness is approximately proportional to the norm of the normalized state vector; while in the other two layers, the thickness tends toward a saturated value as the trajectory moves further from the origin. The feasibility and effectiveness of the proposed approaches are verified through their application to several representative numerical examples.
\end{abstract}

\section{INTRODUCTION}

Sliding mode control is a nonlinear feedback control technique with a proven robustness against modeling uncertainties and external disturbances [12][13][15]. However, as the uncertainties or disturbances in the system increase, a greater control effort is required, and the resulting control signal may induce a chattering phenomenon. Various approaches have been proposed to attenuate this undesirable effect [1]-[11], [13]-[15].

Wang and Chen [14] designed a sliding mode position

Paper submitted 05/24/07; accepted 12/18/07. Author for correspondence: Chyun-Chau Fuh (e-mail:f0005@mail.ntou.edu.tw).

* Department of Mechanical and Mechatronic Engineering, National Taiwan Ocean University, Keelung, Taiwan, R.O.C. controller with an adaptive load torque estimator to control the position of an induction motor in such a way that chattering effects were eliminated. Lo and Kuo [8] proposed a decoupled fuzzy sliding mode controller design in which chattering was avoided by introducing a fuzzy boundary layer at the sliding surface. In [4], Bartolini and Pydynowski presented a method for avoiding chattering by establishing a continuous control effort based on the integral of a discontinuous signal on a suitable manifold in an augmented state space. Bartolini et al. [2][3] presented a solution for eliminating chattering by extending a second-order sliding mode controller from single-input nonlinear systems with particular types of uncertainties to multi-input uncertain nonlinear systems. In [1], the same authors proposed a variable structure control scheme with a reduced boundary layer dimension to preserve the control performance while simultaneously suppressing the chattering phenomenon. Kachroo and Tomizuka [7] reduced chattering by applying continuous control within a boundary layer established at the switching surface. Chen et al. [6] proposed a sliding mode control scheme for uncertain linear systems in which the width of the boundary layer was adjusted on line in accordance with the state norm. It was shown that the state-dependent boundary layer design effectively eliminated chattering while simultaneously ensuring almost perfect control accuracy.

In general, the studies outlined above use one of two different approaches to reduce or eliminate the chattering phenomenon in systems regulated by sliding mode control. In the first approach, a higher-order sliding mode is used to produce a chattering-free control effect. However, this technique is problematic in the sense that the derivative of the state variable can not be measured directly, but must be estimated using an observer variable. In the second approach, a boundary layer is introduced around the sliding surface and a continuous control effect is applied within the boundary layer.

This study proposes four different variable-thickness boundary layers. The thicknesses of the first two boundary layers are approximately proportional to the norm of the normalized state vector, while the thicknesses of the latter two layers gradually tend toward a saturated value as the system trajectory moves further from the origin. The feasibility and effectiveness of the proposed approaches are verified through their application to several numerical examples. 


\section{MAIN RESULTS}

Consider the single-input nonlinear system

$$
x^{(n)}=f(\mathbf{x}, t)+b(\mathbf{x}, t) u(t),
$$

where $\mathbf{x}=\left[x, \dot{x}, \ldots, x^{(n-1)}\right]^{T}$ is the state vector, $u$ is the control input, and $x$ is the output. The nonlinear function $f(\mathbf{x}, t)$ is unknown, but can be estimated as $\hat{f}(\mathbf{x}, t)$. The estimation error on $f(\mathbf{x}, t)$ is assumed to be bounded by some known function $F(\mathbf{x}, t)$, i.e.

$$
|f(\mathbf{x}, t)-\hat{f}(\mathbf{x}, t)|<F(\mathbf{x}, t) .
$$

It is assumed that the aim of the control system is to drive the state vector $\mathbf{x}$ such that it tracks a given desired state vector $\mathbf{x}_{d}=\left[x_{d}, \dot{x}_{d}, \ldots, x_{d}^{(n-1)}\right]^{T}$ despite the presence of uncertainties or disturbances. The error between the current state and the desired state is defined by the vector $\mathbf{e}=\mathbf{x}_{d}-\mathbf{x}=\left[e, \dot{e}, \ldots, e^{(n-1)}\right]^{T}$. The sliding surface has the form

$$
s=\left(\frac{d}{d t}+\lambda\right)^{n-1} e=\sum_{i=1}^{n} C_{i-1}^{n-1} \lambda^{i-1} e^{(n-i)}=0,
$$

where $\lambda$ is a positive constant whose value is specified such that the differential equation $s=0$ is stable. In deriving the sliding mode control law which forces the system trajectory to move along the sliding surface, it is customary to choose a law which satisfies the following sliding condition:

$$
s \dot{s} \leq-\eta|s|,
$$

where $\eta$ is a positive constant. This sliding condition guarantees that $|s|$ decreases if $\mathbf{x}$ is outside the sliding surface, i.e. all of the trajectories outside $s=0$ move toward the surface, while those on $s=0$ remain on the sliding surface. Although a finite time is required for the trajectories to reach the surface, the choice of the sliding surface implies that once the surface has been reached, the error will converge exponentially to zero.

The control law which guarantees satisfaction of this sliding condition is defined in the following theorem.

Theorem 1 [10]: Consider the single-input nonlinear lumped parameter system, affine in control, given in Equation (1), where

$$
0 \leq b_{\min }(\mathbf{x}, t) \leq b(\mathbf{x}, t) \leq b_{\max }(\mathbf{x}, t) .
$$

The control law

$$
u(t)=\hat{b}(x, t)^{-1}[\hat{u}(t)-k(\mathbf{x}, t) \operatorname{sgn}(s)],
$$

where

$$
\begin{gathered}
\hat{u}(t)=\sum_{i=2}^{n} \mathrm{C}_{i-1}^{n-1} \lambda^{i-1} e^{(n-i+1)}+x_{d}^{(n)}-\hat{f}(\mathbf{x}, t) \\
k(\mathbf{x}, t)=\alpha(\mathbf{x}, t)(F(\mathbf{x}, t)+\eta)+(\alpha(\mathbf{x}, t)-1)|\hat{u}(t)|
\end{gathered}
$$

$$
\hat{b}(\mathbf{x}, t)=\sqrt{b_{\max }(\mathbf{x}, t) b_{\min }(\mathbf{x}, t)}
$$

and

$$
\alpha(\mathbf{x}, t)=\sqrt{\frac{b_{\max }(\mathbf{x}, t)}{b_{\min }(\mathbf{x}, t)}},
$$

ensures the invariant condition specified in Equation (4).

Although sliding mode controllers theoretically provide excellent robustness against uncertainties and disturbances, applying a finite sampling frequency when performing digital control causes the state trajectory to pass to the other side of the sliding surface, and this induces chattering in the system. Chattering is highly undesirable since its suppression requires extremely high control activity. Furthermore, chattering may excite high-frequency dynamics neglected in the course of modeling, such as resonant structural modes or actuator time-delays.

The chattering problem is commonly addressed by applying a smooth control interpolation in a boundary layer neighboring the sliding surface. The boundary layer is generally defined by replacing the sign function $\operatorname{sgn}(s)$ in Equation (6) by a saturation function $\operatorname{sat}(s)$, defined as

$$
\operatorname{sat}(s)=\left\{\begin{array}{cl}
1, & \delta<s \\
s / \delta, & -\delta<\mathrm{s} \leq \delta, \\
-1, & s \leq-\delta
\end{array}\right.
$$

where $\delta$ is normally constant and characterizes the error associated with the smooth approximation of the sign function by the saturation function. $2 \delta$ defines the thickness of the boundary layer. In general, the greater the boundary layer thickness, the smoother the control signal. Although introducing a boundary layer alleviates the chattering phenomenon, the sliding condition is no longer guaranteed. In practice, the system state contracts to a small residual set around $\mathbf{e}=\mathbf{0}$ when a boundary layer is introduced. The size of this residual set depends on the thickness of the boundary layer, i.e. the thinner the boundary layer, the smaller the residual set. Consequently, smoothing the control signal through the introduction of a boundary layer at the sliding surface results in a loss of control accuracy.

In an attempt to suppress the chattering phenomenon while preserving the control accuracy, this study proposes four different variable-thickness boundary layers. Since the sliding function is the weighted sum of the terms relating to the tracking error, a large boundary layer thickness does not necessarily imply a large tracking error [6]. In the sliding function, the terms $\lambda^{n-1} e$ and $e^{(n-1)}$ both have a dominant effect. However, the difference between $e$ and $e^{(n-1)}$ may be very large, particularly when $\lambda>>1$ or $\lambda<<1$. Therefore, observing the variation of the trajectory or designing the thickness of the boundary layer based on the error space is inappropriate. Accordingly, this study introduces a normalized error vector, $\mathbf{z}$, expressed as follows: 


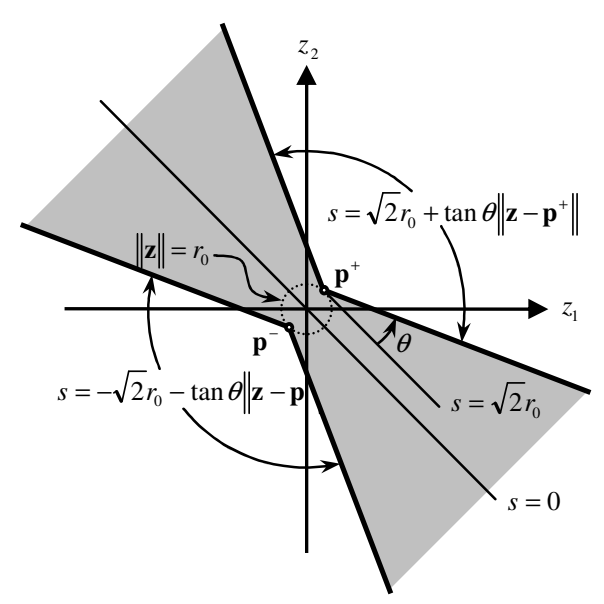

Fig. 1. Schematic description of variable-thickness boundary layer $N_{1}(s)$ for second-order system.

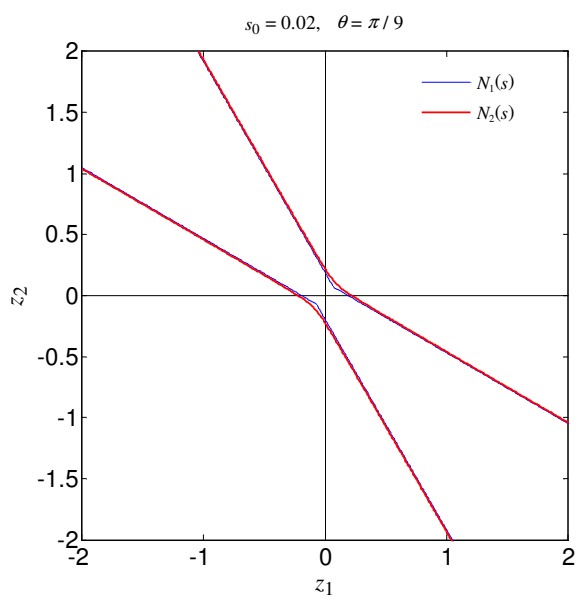

Fig. 2. Comparison of variable-thickness boundary layers $N_{1}(s)$ and $N_{2}(s)$.

$$
\mathbf{z}=\left[z_{1}, z_{2}, \ldots, z_{n}\right]^{T},
$$

where

$$
z_{i}=C_{n-i}^{n-1} \lambda^{n-i} e^{(i-1)} .
$$

The sliding function can then be rewritten in the following normalized form:

$$
s=\sum_{i=1}^{n} z_{i}
$$

In [6], the authors reported that for a sliding mode controller with a small boundary layer thickness, chattering usually occurs only during the transient stage when the system trajectory is far from the origin. As the trajectory approaches the origin, the chattering effect gradually reduces, or even disappears completely. This implies that when the trajectory is far from the origin, a large boundary layer thickness should be used to suppress chattering. Conversely, when the trajectory approaches the

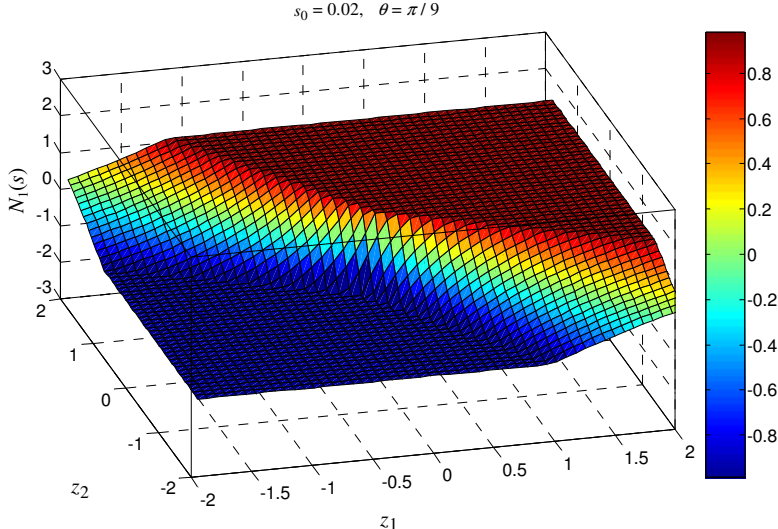

Fig. 3(a). 3-D plot of variable-thickness boundary layer $N_{1}(s)$ for second-order system.

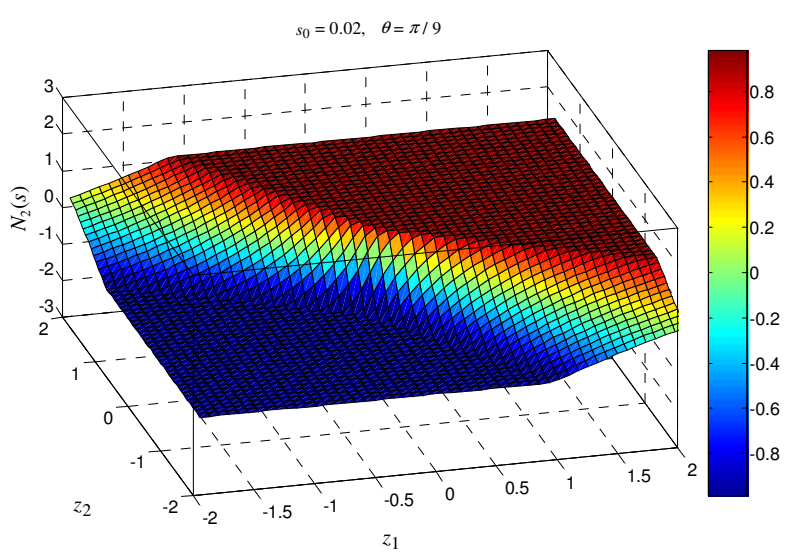

Fig. 3(b). 3-D plot of variable-thickness boundary layer $N_{2}(s)$ for second-order system.

origin, the thickness of the boundary layer should be reduced in order to improve the control accuracy. In other words, the ideal boundary layer thickness varies as a function of the modulus of the system state.

Two constant vectors and three scalars functions are introduced as follows:

$$
\begin{gathered}
\mathbf{p}^{+}=\frac{r_{0}}{\sqrt{n}}[\underbrace{1, \ldots, 1}_{n}]^{T}, \\
\mathbf{p}^{-}=\frac{-r_{0}}{\sqrt{n}}[\underbrace{1, \ldots, 1}_{n}]^{T}, \\
\delta_{1}^{+}=s_{0}+\tan \theta\left\|\mathbf{z}-\mathbf{p}^{+}\right\|, \\
\delta_{1}^{-}=s_{0}+\tan \theta\left\|\mathbf{z}-\mathbf{p}^{-}\right\|, \\
s_{0}=\sqrt{n} r_{0},
\end{gathered}
$$

where $n$ is the system order and $r_{0}$ and $\theta$ are appropriate positive constants. The variable-thickness boundary layer is defined as follows: 


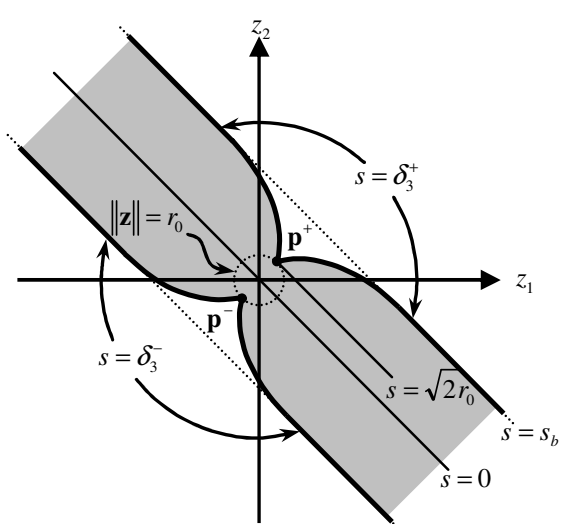

Fig. 4. Schematic description of variable-thickness boundary layer $N_{3}(s)$ for second-order system.

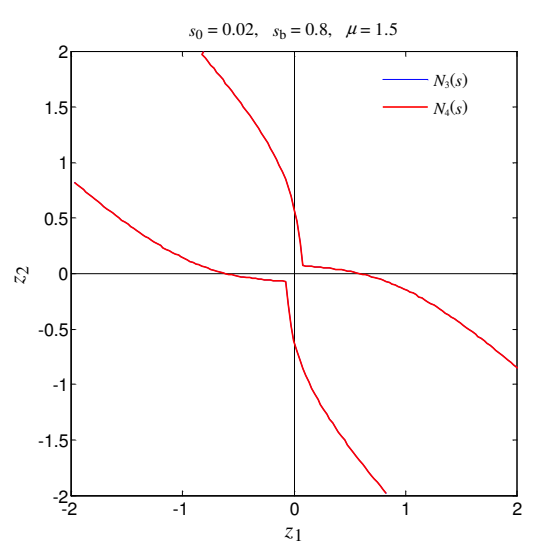

Fig. 5. Comparison of variable-thickness boundary layers $N_{3}(s)$ and $N_{4}(s)$.

$$
N_{1}(s)=\left\{\begin{array}{ll}
1, & \delta_{1}^{+}<s \\
s / \delta_{1}^{+}, & 0<s \leq \delta_{1}^{+} \\
s / \delta_{1}^{-}, & -\delta_{1}^{-}<s \leq 0 \\
-1, & s \leq-\delta_{1}^{-}
\end{array},\right.
$$

and is used to replace the sign function $\operatorname{sgn}(s)$ in the sliding mode control law given in Equation (6).

The variable-thickness boundary layer for a second-order system (i.e. $n=2$ ) is shown schematically in Fig. 1, in which $\mathbf{p}^{+}$and $\mathbf{p}^{-}$are the tip points of the boundary and the distance (in $\mathbf{z}$-coordinates) between the two points is equal to $2 r_{0}$. As shown, the lines $s=\sqrt{2} r_{0}$ and $s=-\sqrt{2} r_{0}$ pass through points $\mathbf{p}^{+}$and $\mathbf{p}^{-}$, respectively. Hence, the smallest thickness (in $s$-coordinates) of the variable-thickness boundary layer is equal to $2 s_{0}$ or $2 \sqrt{2} r_{0}$. The angle between the sliding line $s=0$ and the $z_{1}$-axis is always $\pi / 4$.

Omitting the vectors $\mathbf{p}^{+}$and $\mathbf{p}^{-}$in Equations (7) and (8), $\delta_{1}^{+}$becomes $\delta_{1}^{-}$, and hence it can be shown that

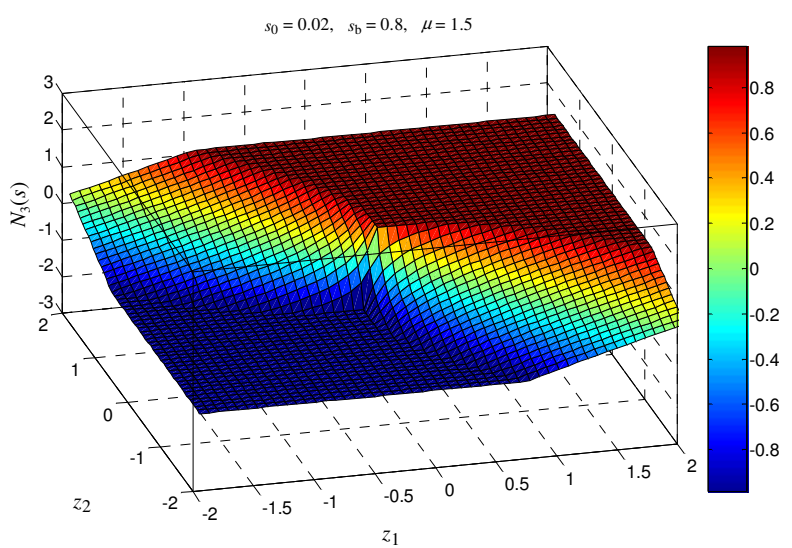

Fig. 6(a). 3-D plot of variable-thickness boundary layer $N_{3}(s)$ for second-order system.

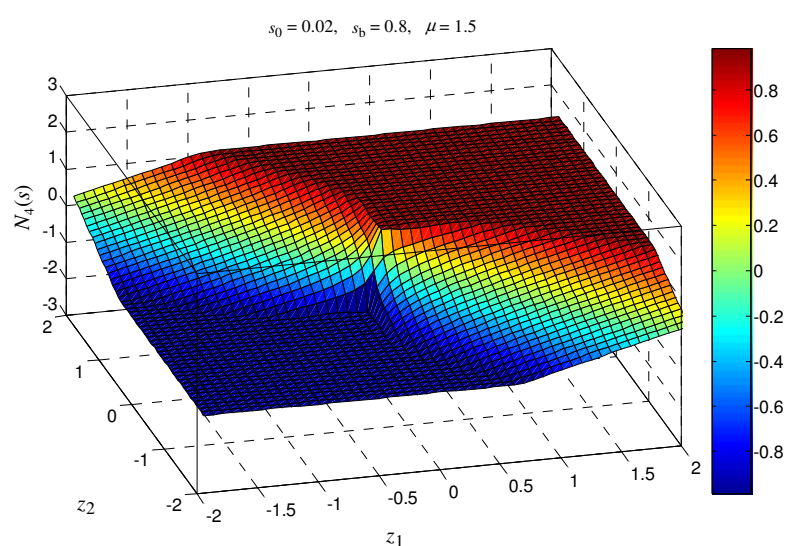

Fig. 6(b). 3-D plot of variable-thickness boundary layer $N_{4}(s)$ for second-order system.

$$
\delta_{2}=s_{0}+\tan \theta\|\mathbf{z}\|
$$

The variable-thickness boundary layer given in Equation (20) can then be approximated as

$$
N_{2}(s)= \begin{cases}1, & \delta_{2}<s \\ s / \delta_{2}, & -\delta_{2}<s \leq \delta_{2} . \\ -1, & s \leq-\delta_{2}\end{cases}
$$

Fig. 2 compares boundary layers $N_{1}(s)$ and $N_{2}(s)$ for a second-order system. As shown, $N_{1}(s)$ consists of four straight lines, while $N_{2}(s)$ has the form of a hyperbola. Figs. 3(a) and 3(b) present three-dimensional representations of $N_{1}(s)$ and $N_{2}(s)$. Although the two plots are very similar, a close inspection shows that $N_{1}(s)$ is not smooth, i.e. it has two tip points near its narrowest region. By contrast, $N_{2}(s)$ is smooth over the entire surface. However, in general, it is apparent that $N_{2}(s)$ provides a good approximation of $N_{1}(s)$.

In $N_{1}(s)$ and $N_{2}(s)$, the larger the value of $\|\mathbf{z}\|$, the greater the thickness of the boundary layer. However, a thicker boun- 


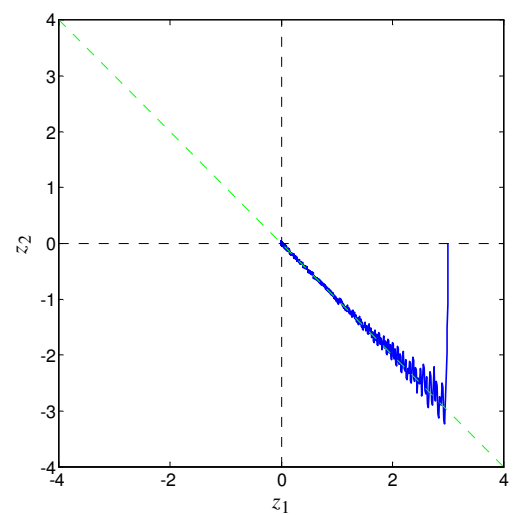

Fig. 7. Phase trajectory of system using sliding mode controller without boundary layer.
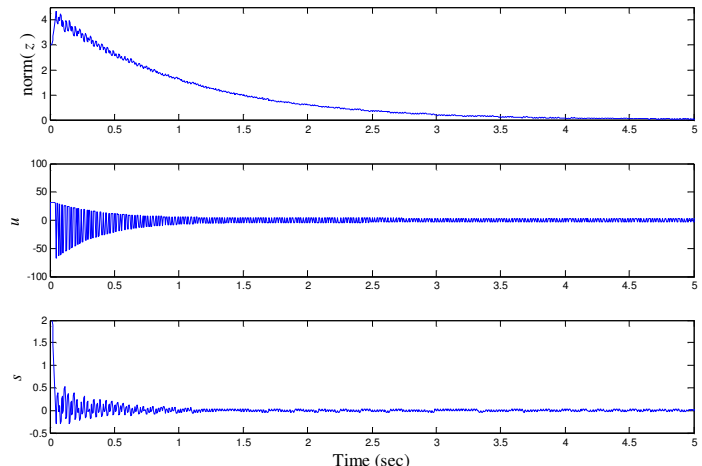

Fig. 8. Time responses of system using sliding mode controller without boundary layer.

dary layer implies a reduced robustness against uncertainty or disturbance. Therefore, the thickness of the boundary layer should be constrained. Accordingly, this study proposes a variable-thickness boundary layer with a limited thickness. The following scalar functions are defined:

$$
\begin{aligned}
& \delta_{3}^{+}=s_{0}+\left(s_{b}-s_{0}\right) \frac{2}{\pi} \tan ^{-1}\left(\mu \sqrt{\left\|\mathbf{z}-\mathbf{p}^{+}\right\|^{2}-\left(\left(\mathbf{z}-\mathbf{p}^{+}\right) \cdot \mathbf{n}\right)^{2}}\right), \\
& \delta_{3}^{-}=s_{0}+\left(s_{b}-s_{0}\right) \frac{2}{\pi} \tan ^{-1}\left(\mu \sqrt{\left\|\mathbf{z}-\mathbf{p}^{-}\right\|^{2}-\left(\left(\mathbf{z}-\mathbf{p}^{-}\right) \cdot \mathbf{n}\right)^{2}}\right),
\end{aligned}
$$

where $\mathbf{p}^{+}, \mathbf{p}^{-}$and $s_{0}$ are defined in Equations (15), (16) and (19), respectively; $\mathbf{n}=\frac{1}{\sqrt{n}}[\underbrace{1, \ldots, 1}_{n}]^{T}$ is the unity normal vector of the sliding surface $s$; the dot denotes an inner product; $2 s_{b}$ is the saturated thickness of the boundary layer; and $\mu$ is a positive constant. The variable-thickness boundary layer with a limited thickness is then defined as

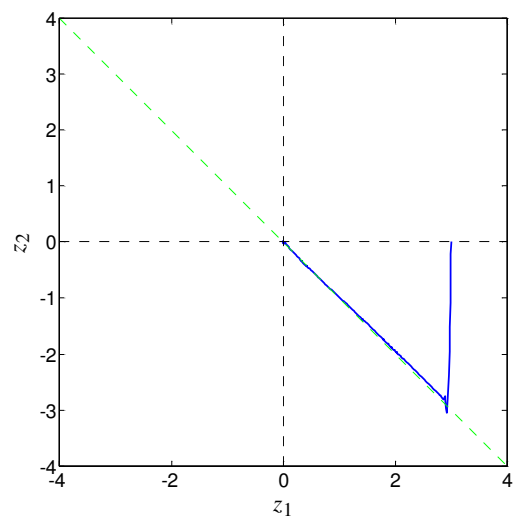

Fig. 9(a). Phase trajectory of system using sliding mode controller with constant-thickness boundary layer.

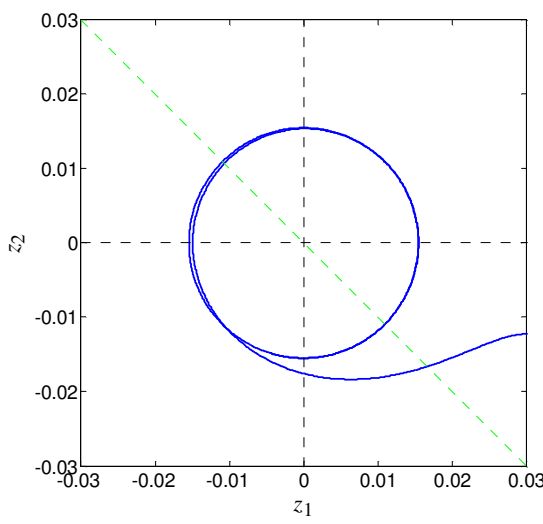

Fig. 9(b). Phase trajectory near origin of system using sliding mode controller with constant-thickness boundary layer.

$$
N_{3}(s)=\left\{\begin{array}{ll}
1, & \delta_{3}^{+}<s \\
s / \delta_{3}^{+}, & 0<s \leq \delta_{3}^{+} \\
s / \delta_{3}^{-}, & -\delta_{1}^{-}<s \leq 0 \\
-1, & s \leq-\delta_{3}^{-}
\end{array} .\right.
$$

Fig. 4 presents a schematic illustration of $N_{3}(s)$. The thickness of the boundary layer in the narrowest region is equal to $2 \sqrt{2} r_{0}$ and the range of the narrow band is inversely proportional to the parameter $\mu$.

As in the derivations for boundary layers $N_{1}(s)$ and $N_{2}(s)$, the variable-thickness boundary layer given in Equation (25) can be simplified to

$$
N_{4}(s)= \begin{cases}1, & \delta_{4}<s \\ s / \delta_{4}, & -\delta_{4}<s \leq \delta_{4}, \\ -1, & s \leq-\delta_{4}\end{cases}
$$

where

$$
\delta_{4}=s_{0}+\left(s_{b}-s_{0}\right) \frac{2}{\pi} \tan ^{-1}\left(\mu \sqrt{\|\mathbf{z}\|^{2}-\frac{s^{2}}{n}}\right) .
$$




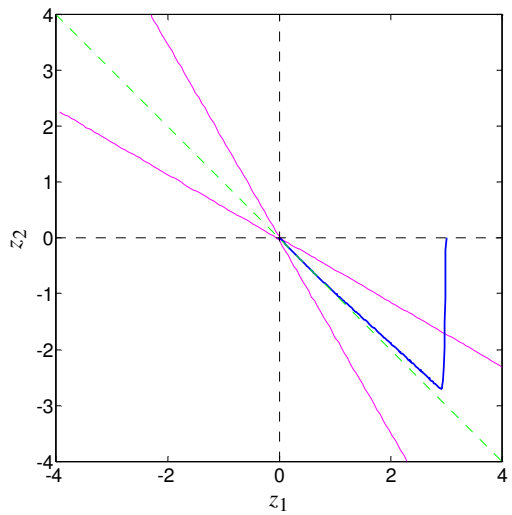

Fig. 10(a). Phase trajectory of system using sliding mode controller with variable-thickness boundary layer $N_{2}(s)$.

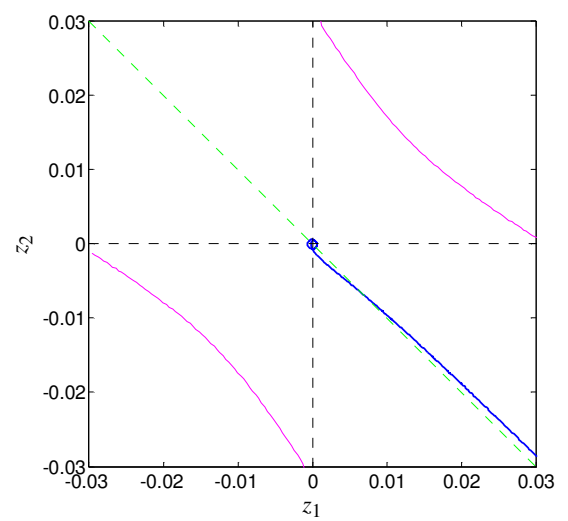

Fig. 10(b). Phase trajectory near origin of system using sliding mode controller with variable-thickness boundary layer $N_{2}(s)$.

Fig. 5 compares boundary layers $N_{3}(s)$ and $N_{4}(s)$ for a second-order system. It can be seen that the two layers are virtually superimposed one on top of each other. Figs. 6(a) and 6(b) present three-dimensional representations of $N_{3}(s)$ and $N_{4}(s)$, respectively. There is no discernible difference between the two surfaces, and hence it can be inferred that $N_{4}(s)$ provides a good approximation of $N_{3}(s)$.

\section{NUMERICAL SIMULATIONS}

The following simulation examples demonstrate the effects of sliding mode control achieved using different types of boundary layer. The simulations consider the following second-order nonlinear dynamic system:

$$
\begin{aligned}
& \dot{x}_{1}=x_{2} \\
& \dot{x}_{2}=a_{1} x_{1}+a_{2} x_{1}^{3}+a_{3} x_{2}+d(t)+b u
\end{aligned}
$$

where the parameters are unknown, but their bounds can be estimated in advance. The actual values of the parameters used in the simulations are $a_{1}=1, a_{2}=-1, a_{3}=-0.25$, $d(t)=0.3 \sin (t)$ and $b=2$, respectively. An assumption is

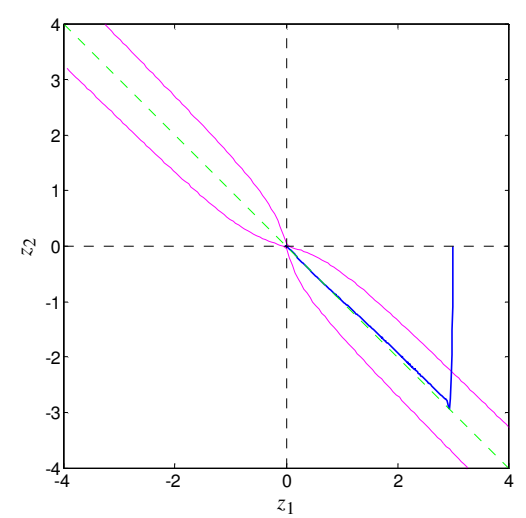

Fig. 11(a). Phase trajectory of system using sliding mode controller with variable-thickness boundary layer $N_{4}(s)$.

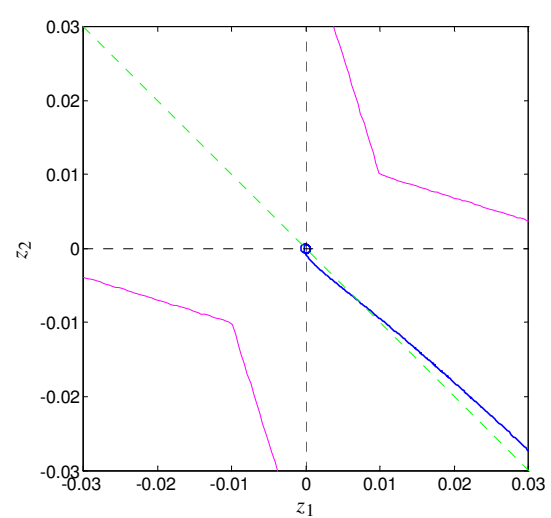

Fig. 11(b). Phase trajectory near origin of system using sliding mode controller with variable-thickness boundary layer $N_{4}(s)$.

made that the parameters and their bounds are estimated to be

$$
\begin{aligned}
& \hat{a}_{1}=1, \hat{a}_{2}=-1.5, \hat{a}_{3}=-0.25, \hat{d}(t)=0, \hat{b}=2 \\
& a_{1} \in[0.6,1.4], a_{2} \in[-2.5,-0.5], a_{3} \in[-0.5,0], \\
& |d(t)|<0.4, \text { and } b \in[1,4] .
\end{aligned}
$$

Thus, it can be estimated that the error on $f(\mathbf{x}, t)$ is bounded by

$$
|f(\mathbf{x}, t)-\hat{f}(\mathbf{x}, t)|<F(\mathbf{x}, t)=0.4\left|x_{1}\right|+\left|x_{1}\right|^{3}+0.25\left|x_{2}\right|+0.4 .
$$

It is assumed that the aim of the control system is to regulate the state vector to zero, i.e. $\mathbf{x}_{d}=\left[x_{d}, \dot{x}_{d}\right]=\mathbf{0}$. The error vector is defined as $\mathbf{e}=[e, \dot{e}]=\left[-x_{1},-x_{2}\right]$.

The sliding function is specified as

$$
s=\dot{e}+\lambda e,
$$

where $\lambda>0$ such that the differential equation $s=\dot{e}+\lambda e=0$ is stable. The following control law is chosen to satisfy the reaching condition of sliding mode control scheme:

$$
u(t)=0.5[\hat{u}(t)-k(\mathbf{x}, t) \operatorname{sgn}(s)],
$$

where 


$$
\begin{gathered}
\hat{u}(t)=\lambda \dot{e}+\ddot{x}_{d}-\hat{f}(\mathbf{x}, t)=\lambda \dot{e}+\ddot{x}_{d}-x_{1}+1.5 x_{1}^{3}+0.25 x_{2}, \\
k(\mathbf{x}, t)=2(F(\mathbf{x}, t)+\eta)+|\hat{u}(t)| .
\end{gathered}
$$

The controller parameters are specified as $\lambda=1$ and $\eta=3$, and the sampling time is $0.005 \mathrm{sec}$.

The trajectory and time response of the system governed by the control law given in Equation (31) are shown in Figs. 7 and 8 , respectively. The results show that when a boundary layer is not employed, a violent chattering effect takes place when the system trajectory is far from the origin. However, it is observed that the chattering phenomenon is gradually alleviated as the trajectory approaches the origin.

In the second simulation, the sign function $\operatorname{sgn}(s)$ in the power law given in Equation (31) is replaced by the saturation function $\operatorname{sat}(s)$ defined in Equation (11), and the value of $\delta$ is adjusted to suppress the chattering phenomenon. Fig. 9(a) shows the phase trajectory of the controlled system for the case of $\delta=0.5$. Fig. 9(b) illustrates the system trajectory near the origin. It is observed that the trajectory wanders as a result of the time-varying disturbance $d(t)$. To contract the system state to a smaller residual set around the origin, it is necessary to reduce the thickness of the constant boundary layer. However, chattering then occurs during the transient stage when the system trajectory is far from the origin. In this case, the minimum boundary layer thickness which suppresses chattering completely is approximately $2 \delta=1.0$.

In the third simulation, the sign function $\operatorname{sgn}(s)$ in the control law given in Equation (31) is replaced by the variable-thickness boundary layer $N_{2}(s)$ defined in Equation (22), with values of $s_{0}=0.02$ and $\theta=\pi / 9$. The corresponding control results are shown in Figs. 10(a) and 10(b). It can be seen that the chattering phenomenon almost disappears and the system trajectory contracts to a very small residual set around the origin. (Note that the control results obtained using the variable-thickness boundary layer $N_{1}(s)$ are similar to those shown for $N_{2}(s)$ and are therefore not presented here.)

In the final simulation, the sliding mode controller applies the variable-thickness boundary layer $N_{4}(s)$ defined in Equation (26), where $\delta_{4}$ is simplified to

$$
\delta_{4}=s_{0}+\left(s_{b}-s_{0}\right) \frac{2}{\pi} \tan ^{-1}\left(\frac{\mu}{\sqrt{2}}\left|z_{1}-z_{2}\right|\right),
$$

with $s_{0}=0.02, s_{\mathrm{b}}=0.8$ and $\mu=1.5$. The results for the system trajectory are shown in Figs. 11(a) and 11(b). It is observed that the chattering phenomenon is significantly reduced and the system trajectory contracts to a very small range around the origin. (The control results obtained using the variable-thickness boundary layer $N_{3}(s)$ are similar to those of $N_{4}(s)$ and are therefore omitted here).

\section{CONCLUSION}

In attempting to obtain a compromise between the requirement for control accuracy and the desire to eliminate the chattering phenomenon, this paper has proposed four different variable-thickness boundary layers, i.e. $N_{1}(s), N_{2}(s), N_{3}(s)$, and $N_{4}(s)$, by replacing the sign function $\operatorname{sgn}(s)$ in the original sliding mode control law with appropriate boundary layer functions. In $N_{1}(s)$ and $N_{2}(s)$, the higher the norm of the normalized state vector $\|\mathbf{z}\|$, the greater the thickness of the boundary layer. In $N_{3}(s)$ and $N_{4}(s)$, both the thickness and the range of the narrowest region in the boundary layer are adjustable, and the boundary layer thickness gradually tends toward an adjustable constant when the system trajectory is far from the origin. The feasibility and effectiveness of the proposed approaches have been verified in a series of numerical examples.

\section{REFERENCES}

1. Bartolini, G., Ferrara, A., and Usai, E., "On boundary layer dimension reduction in sliding mode control of SISO uncertain nonlinear systems," IEEE International Conference on Control Applications, Vol. 1, pp. 242 - 247 (1998).

2. Bartolini, G., Ferrara, A., and Usai, E., "Chattering avoidance by second-order sliding mode control," IEEE Transactions on Automatic Control, Vol. 43, No. 2, pp. 241 - 246 (1998).

3. Bartolini, G., Ferrara, A., Usai, E., and Utkin, V. I., "On multi-input chattering-free second-order sliding mode control," IEEE Transactions on Automatic Control, Vol. 45, No. 9, pp. 1711 - 1717 (2000).

4. Bartolini, G. and Pydynowski, P., "An improved chattering free VSC scheme for uncertain dynamical systems," IEEE Transactions on Automatic Control, Vol. 41, No. 8, pp. 1220-1226 (1996).

5. Bondarev, A. G., Bondarev, S. A., Kostyleva, N. E., and Utkin, V. I., "Sliding modes in systems with asymptotic state observers," Automatic and Remote Control, Vol. 46, No. 6, pp. 49-64 (1985).

6. Chen, M.-S., Hwang, Y.-R., and Tomizuka, M, "A state-dependent boundary layer design for sliding mode control," IEEE Transactions on Automatic Control, Vol. 47, No. 10, pp. 1677-1681 (2002).

7. Kachroo, P. and Tomizuka, M., "Chattering reduction and error convergence in the sliding mode control of a class of nonlinear systems," IEEE Transactions on Automatic Control, Vol. 41, No. 7, PP. 1063-1068 (1996).

8. Lo, J.-C. and Kuo, Y.-H., "Decoupled fuzzy sliding-mode control," IEEE Transaction on Fuzzy Systems, Vol. 6, No. 3, pp. 426-435 (1998).

9. Slotine, J. J., "Sliding controller design for nonlinear systems," International Journal of Control, Vol. 40, No. 2, pp. 421-434 (1984).

10. Slotine, J. J. and Li, W, Applied nonlinear control, Englewood Cliffs, NJ: Prentice-Hall (1991).

11. Slotine, J. J. and Sastry, S. S., "Tracking control of non-linear systems using sliding surfaces, with application to robot manipulators," International Journal of Control, Vol. 38, No. 2, pp. 465-492 (1983).

12. Utkin, V. I. "Variable structure systems with sliding modes," IEEE Transactions on Automatic Control, Vol. 22, No. 2, pp. 212-222 (1977).

13. Utkin, V. I., "Application oriented trends in sliding mode control theory," International Conference on Industrial Electronics, Control, and Instrumentation, Vol. 3, pp. 1937 - 1942 (1993).

14. Wang, W.-J., and Chen, J.-Y., "A new sliding mode position controller with adaptive load torque estimator for an induction motor," IEEE Transaction on Energy Conversion, Vol. 14, No. 3, pp. 413-418 (1999).

15. Young, K. D., Utkin, V. I., and Ozguner, U., “A control engineer's guide to sliding mode control," IEEE Transactions on Control Systems Technology, Vol. 7, No. 3, pp. 328 - 342 (1999). 